Die Funktion des komplexen Wiederkäuermagens leitet P. einmal aus den Resultaten der anatomischen und geweblichen Untersuchungen ab. Sodann stützte er sich, ähnlich wie es WEPFER getan hatte, auf die Beschaffenheit des Nahrungsbreies in den einzelnen Mägen geschlachteter Tiere, die Rückschlüsse auf die Funktion der einzelnen Magenabschnitte zu ziehen erlaubte. Überraschend gut gelingt ihm dabei die Unterscheidung der mechanischen Funktion der Vormägen von der chemischen Einwirkung des Labmagens.

CoLE schreibt in seiner Geschichte der vergleichenden Anatomie ${ }^{39}$, daß das Werk P.s über den Wiederkäuermagen als eine der besten früheren Bearbeitungen dieses Gebietes zu betrachten sei. Es war also wohl der Mühe wert, dieses umfangreichste, auf sorgfältigen Untersuchungen aufgebaute und mit erschöpfender Angabe der früheren Literatur versehene Werk Peyers in Erinnerung zu rufen.

\title{
Prognostikation auf 24 zukünftige Jahre von Theophrastus Paracelsus und ein zeitgenössischer Deutungsversuch
}

Von Bernhard Milt, Zürich

Vor etlichen Jahren erhielt der Verfasser dieser Zeilen einen Brief aus Frankreich von einem Unbekannten, mit der Anfrage, ob es richtig sei, daß diese Prognostik Theophrasts gerade die Zeit nach dem letzten Kriegsende im Auge gehabt habe. Es muß damals in unserm Nachbarland eine Schrift erschienen sein, welche eben dieses behauptete. Das Schriftchen konnte durch unsern Buchhandel nicht aufgetrieben werden, und so hat der Schreiber seinen Titel und Verfasser längst vergessen. Ihn interessierte in der Folge mehr die kleine Arbeit von Paragelsus, die nach mehr als vier Jahrhunderten aktuell sein sollte.

Sudhoff hat sie in den 10. Band seiner Paracelsus-Ausgabe aufgenommen; er bezeichnete sie einmal als politisch-mantische, ein andermal als religionspolitisch-philosophische Arbeit, in der Einleitung (S. XLII) auch als politische Voraussage. Über Inhalt und Sinn des Werkleins war er sich offenbar nicht völlig klar. Der genauere Titel lautet:

39 J.F. CoLe, A History of comparative Anatomy, London 1944, S. 251. 
Prognostikation auf XXIIII. jar zukünfftig/durch den hochgelerten Doctorem Paracelsum / Geschriben zu dem Großmechtigsten/Durchleuchtigsten Fürsten und Herren/Herren Ferdinanden etc. Römischen Künig/ Ertzhertzog etc. zu Osterreych etc. Getruckt zu Augspurg durch Heynrich Steyner/am XXIII. tag Augusti/Anno/MDXXXVI.

Sudhoff gibt an, daß diese deutsch herausgegebene Schrift drei Tage später auch in lateinischer Fassung erschienen sei, durch den gewandten Humanisten Margus Tatius Alpinus, den spätern Poesieprofessor an der Hochschule von Ingolstadt. Die lateinische Fassung soll drei Ausgaben erlebt haben, die deutsche wesentlich mehr, bis weit ins 17. Jahrhundert hinein.

Sich mit dem Sinn dieser in sibyllinischen Ausdrücken verfaßten Prognostikation auseinanderzusetzen, würde sich der Verfasser dieser Zeilen kaum berufen gefühlt haben, hätte ihm nicht der Zufall ein altes Exemplar aus dem Jahr 1536 in die Hände gespielt, mit zeitgenössischen oder wenig spätern Randnotizen von einem unbekannten Autor. Sie sind zwar nicht vollständig erhalten. Irgendein früherer Besitzer ließ das kleine Büchlein ThEoPHRASTS mit einer andern mantischen Schrift von etwas kleinerem Format zusammenbinden, wobei dann die Ränder des größern beschnitten und nicht wenige Randbemerkungen zerstört oder verstümmelt wurden. Das noch übrig gebliebene genügt dennoch für einen Eindruck, wie sich der damalige Besitzer unseres Exemplars (Ms. XVIII-158, 1 der Zürcher Zentralbibliothek) seinen Inhalt erklärt hat. Die folgenden Blätter wollen nun anhand dieser Randbemerkungen des Unbekannten eine Deutung dieser Prognostikation versuchen.

Die Prognostik selbst ist eingerahmt von einem Vorwort und einer Nachrede mit erklärenden Bemerkungen von Paracelsus selber. Die Prognostikation oder Weissagung besteht aus 32 Holzschnitten, deren jeder mit seinem zugehörigen kurzen Text je eine Seite füllt. Auf den nachfolgenden Blättern bedeutet $B=$ Bild, $T=$ Text und $K=$ Kommentar bzw. Randnotizen des Unbekannten, zu denen wegen ihrer fragmentarischen Form teilweise Konjekturen nötig waren.

Paracelsus beginnt mit der Feststellung, daß wir von irdischen Dingen kein großes Wissen haben, daß wir immer wieder straucheln und anstoßen. Wie viel weniger können wir über das Überirdische Aussagen machen und Kenntnisse von Gestirnen und ihren Wirkungen besitzen, da wir doch im Himmel weder mit unsern Augen noch gar mit unsern Füßen Erfahrungen 
sammeln können. Da uns Gott so geschaffen hat, wie wir sind, muß schon er selber unser Lehrmeister sein, da Menschen eine solche Auf gabe nicht übernehmen können. Wer kann ergründen, wie einst die Buchstaben erfunden wurden? Sicher allein durch göttlichen Unterricht. Als es Gott gut fand, daß die Menschen diese Kunst kannten, erlernten sie dieselbe. Gott, der Schöpfer aller Wunderwerke der Natur, schuf auch die Schule, in der wir sie erkennen lernen, viele Dinge, die nicht am Tage liegen. Fängt nicht der Fischer tausend Klafter unter der Wasseroberfläche Fische, die er nie zuvor erblickt hat? Der Bergmann fördert Gold, das tief in der Erde liegt, in die er nicht sehen kann. Gott war beider Lehrmeister. Es gibt keine Heimlichkeit am Firmament, im Moor und unter der Erde, von welcher der Mensch keine Kunde erhalten könnte. Wer will mit dem Finger auf den ersten Lehrmeister deuten und ihn mit Namen nennen? Wohl schafft er im Menschen, ist aber nicht von menschlicher Natur; er unterweist das Menschengeschlecht in den Wundern der Schöpfung nach Gottes Willen.

Den Weltenlauf für 24 Jahre vorauszubestimmen, ist eine schwierige Aufgabe. Des Menschen Tage sind gezählt und abgekürzt, weil er Gott vergessen und so gar nicht nach seinem Willen gelebt hat. Die Menschen tun sich viel Leid an; einer vergönnt dem andern die Sonne. Eine Botschaft findet man nur in den Zeichen von Sonne, Mond und Sternen; den Weg auf Erden kann man nur am Himmel lernen.

Es ist eine einzige Zahl, in der wir auf Erden wandeln sollen, im Eins; mehr sollen wir nicht zählen. Die Gottheit ist zwar eine Dreiheit; aber sie ist ins Eins eingegangen. Da Gott sich selber gewandelt hat und nur noch einer ist, müssen wir Menschen ihm folgen, in dieses Eins uns ergeben und in ihm bleiben. In dieser Zahl ist Ruhe und Frieden und sonst in keiner andern. Was mehr ist, das bedeutet Unruhe und Zank, Streit des einen wider den andern. Wenn ein Rechner eine eigene Zahl setzen will, wer bestimmt dann die Häupter, wer die Größe der Zahl ? Das ist die Not und der Wurm, der an uns nagt. Wie fröhlich und gut ist es, im Eins zu wandeln. Hat doch auch das Firmament seinen natürlichen Lauf in der Zahl, die Erde und alles. Da sich die Menschen in ihrer Vermessenheit über die ihnen gesetzte Zahl Eins erhoben, werden ihnen Zeichen in Sonne, Mond und Sternen vorgehalten. Das Elend fängt aber erst an und endet noch nicht. Selig, wer nicht auf dem Stuhl der Pestilenz sitzt und bei den Sündern wohnt; denn diese werden heimgesucht werden. Jeder mag in seinem eigenen Gewissen erforschen, wo er Platz genommen hat. Wider Gott kann keiner bestehen, keiner ihn überwinden. Da niemand den Sieg wider ihn 
davontragen kann, ist es da nicht eine große Torheit der Menschen, sich gegen ihn aufzulehnen? Sie werden sich am Ende alle krümmen, und wer sollte deshalb traurig sein, daß sich das Ende von Hoffart und Übermut nähert? Wer sich nicht freuen, daß der Hüter der Zahl Eins sich erhebt und dieses Eins zum Gebäude errichtet? Wie ruhig wird es sein, wenn man nichts mehr hinzuzählen muß. Dann wird der Segen von Gott aus gehen. Das Ende wird kommen, und wir werden sein Kommen sehenden Auges nicht gewahren. Keiner soll bekehrt, keiner seinem Urteil entzogen werden. Und niemand soll gegen niemanden einen Argwohn hegen, bis das Ende der Dinge sein Zeugnis ablegt.

Die Zeichen am Himmel zeigen an, daß das Ende nahe ist. Schon vor manchem Jahr wurde die Weltenwende vorausgesagt. Die Zeichen zeigten aber nur den Beginn der Endzeit an. Jetzt ist das Ende nahe; die Tempora nationum sind bald vorbei. Damit der Gerechte nicht weiter verführt werde, muß der Bruch geschehen. Wenn im Himmel kein Weinen war, als Luzifer in den Abgrund der Hölle gestoßen wurde, warum sollten wir traurig sein, wenn sich der Sturz der Hoffart ankündet? Die folgenden 32 Artikel werden sich alle in den kommenden 24 Jahren erfüllen; nach viel Elend werden dann alle zur Demut gebracht sein.

Der Kommentator fügte bei: «In den Tagen der Stimme des siebenten Engels, wenn er posaunen wird, da ist das Geheimnis Gottes vollendet, wie er seinen Knechten, den Propheten, verkündet hat», nach Apokalypse, 10, 7. Als Verbum abbreviatum zitierte er die Worte der Vulgata: «Sed in diebus vocis angeli septimi, cum coeperit tuba canere, consummabitur mysterium Dei, sicut evangelizavit per servos suos Prophetas.»

Die Prognostikation schildert nun in 32 Artikeln, in Bildern mit zugehörigem sibyllinischem Text, den Verlauf der 24jährigen Endzeit.

$B$ I: Ein aus Wolken kommender Arm trägt senkrecht ein zweischneidiges Schwert. Dieses wird von einer Schlange umzingelt, die eine Rute im Maul trägt. Ihr Schwanz liegt in der Öffnung eines von zwei danebenstehenden großen runden Steinen, (oder Rädern ?). T: Das Inwendige der Dinge erkennt man aus äußern Zeichen (Signaturenlehre). Die Schlange ist die Signatur dessen, der mit dem Bild gemeint ist. Denn dieser ist ein Fresser, der alles auffrißt, was in seine Nähe kommt. Er berückt die sich ihm nahenden Menschen nicht mit seiner Frömmigkeit, sondern mit seinem Auftreten, seiner «hüpsche». Selig, der unbefleckt bleibt von ihm; denn keinem läßt er seine Ruhe. K: Der schwertbewehrte Arm ist Cherubim, die Schlange LUTHER. 
B II: Ein Dornbusch, der Lilienembleme trägt. $T$ : Die Magie hat ihn auch mit einer Blume verglichen, die heute schön ist und morgen verdorrt. Er ist nicht in der Weisheit und Gottesfurcht geblieben, die ihn erhalten hätten. Seine eigene Weisheit hat ihn verführt. $K$ : LuthERs Hoffart und Vermessenheit.

B III: Stadt, vor der zwei Haufen Spieße aufragen. $T$ : Du warst unbewußt in schöner Ruhe und kamst in Bewegung, wobei dich dein Übermut einen falschen Weg gehen ließ. Was einst an dir rühmenswert war, dessen kannst du dich nicht mehr rühmen. Dabei hättest du der ganzen Welt helfen können. K: Luthers guter Anfang und seine Trennung.

$B I V$ : Landschaft mit einem der Länge nach gespaltenen Kardinalskreuz, dessen Arme nun, Wegweisern gleich, nach entgegengesetzten Richtungen weisen. T: Du hast die Pflicht gespalten in Rechts und Links. Dein Übermut wird dir $\mathrm{Ha} 3$ bringen. Nur eine Hure buhlt nach zwei Seiten, und auch sie erreicht ihr Lohn. Obschon du jetzt stolz darauf bist, ohne Oberhaupt zu sein, wird dir doch ein Haupt aufgesetzt, wirst du doch zum Glied werden. $K$ : Zweierlei Rechte und zweierlei Pflichten. Ein jeder nach seinem Kopf, sic volo, sic jubeo, so will ich, so befehle ich.

$B V$ : Krone, von einem runden Stein (Mühlstein ?) überhöht; sie scheint im Wasser zu versinken. $T$ : Du hast die Weisheit deiner Krone nicht besessen; darum wird ein Stein über dich fallen und dich formen nach seinem Willen. Du hast unbillig Blut vergossen und den verachtet, der dich von Süden und Osten her heimsuchen wird, ehe du auf zwei gezählt hast. Deine Zuflucht kann dir nicht helfen, da sie selber fällt. $K$ : Schwärmer- und Täuferblut hast du vergossen. Der Kaiser von Österreich wird dich heimsuchen, und die Reichsstädte können dich nicht mehr beschirmen.

$B$ VI: Gebirgige Landschaft, mit einer Blume im Vordergrund. T: Dein starker Geruch ist nicht jedem angenehm; man wird dich deshalb hindern, zur Frucht zu kommen. Du wirst einem weichen müssen, den du noch nicht kennst. Kühn als ein Held bist du am Anfang erstanden. Aber deine Gesellen teilten sich, und was sich teilt, hat keinen Bestand. Ein Lob und ein Ruhm wird dir aber doch bleiben. K: Das Papsttum wird dich nicht zur Frucht kommen lassen; doch deine Selbsterkenntnis bleibt dein Lob und Sieg.

$B$ VII: Ein Priester in Ornat, mit der Inful bedeckt, versinkt in den Wellen, seine Hände nach Hilfe ausstreckend. $T$ : Dein Eigensinn ist schuld daran, daß du von so viel Elend umgeben bist. Die Magie hat dich in einem Stein präformiert, aber du hast dich nicht erkannt. Dein Reich wird zer- 
fallen wie die andern Reiche. Beispiele konnten dich nicht belehren, und deine Weisheit wurde zur zeitlichen Torheit. K: Gregor DER Grosse im Stein. Das Papsttum wurde durch dich geschändet.

$B$ VIII: Das senkrecht gehaltene Schwert in der Hand eines aus Wolken kommenden Arms reicht bis zur Sonne; auf dem Knauf liegt eine Krone. $T$ : Die Stunde kommt, da deine Stärke, deine Bündnisse, deine Macht in sich zusammenfallen. Du selbst wirst zu Boden gehen und mit dir deine Verführten. Denn es ist einer, der austeilen kann, wem und wann er will; alles steht in Gottes Hand. $K$ : Luthertum.

$B I X$ : Ein Löwe springt gegen zwei gespannte Seile. $T: \mathrm{Ob}$ man dich schon fürchtet, ist doch deine Rute schon bereit, mit der dein Rücken geschlagen wird. Hättest du doch Maß gehalten und das Ende bedacht, statt Schein für Sein zu nehmen. Du und dein Anhang werdet lahm und krumm werden; gegenseitig könnt ihr euch dann euer Leid klagen. $K$ : Landgrafen und Kurfürsten fürchten dich. Den Buchstaben hast du für Geist und so Schein für Sein gehalten.

$B X$ : Auf einem Holzsteg im Wasser kniet ein Mann; er schlägt mit einer Holzschaufel (?) auf einen gewölbten Gegenstand (Leder ?). T: Dein Äußeres ist nicht glänzend, als Zeichen für dein Inneres. Dir wurde mehr gedient, als dir gebührt. In deinem Übermut hast du eine Stellung eingenommen, die nicht dir, sondern einem andern zukommt. $K$ : Luther und seine Prädikanten sind nicht hochzeitlich angezogen. Die Umgebung, die ihn groß machte, ist die deutsche Nation. Buchstabe und Schrift sollen allein zur Ehre Gottes gebraucht werden.

$B X I$ : Ein Bär saugt trübselig und allein an seiner Pfote. T: Du hast, als es dir gut ging, das Ende nicht bedacht, den Winter nicht, da es keine Honigwaben mehr gibt. Drum mußt du, einem Bären gleich, an deiner eigenen Pfote saugen. $K$ : Der Bären Art ist grimmig. Luther hat viel Glück gehabt, mehrere Jahre hintereinander.

$B$ XII: Ein umgestürzter Stuhl, darunter die Worte SPR. T: Du hast dich auf einen Stuhl gesetzt, auf den du nicht gehörst (Sankt Petri Stuhl in Rom). Als Bürde und Joch wirst du empfunden und schließlich unter den Stuhl kommen. K: Auf diesen Stuhl gehört ein Apostel, und der Papst wird seinen Sitz wieder einnehmen.

B XIII: Ein Landsknecht zieht mit gezücktem Schwert durchs Land. $T$ : Glück bringt Übermut; drum geht es dir wie Gras, das zu schnell wächst: es wird abgemäht. Das Glück hat dich verblendet und damit dein Schicksal und Ende bestimmt. K: Des Luthertums Hoffart. 
B XIV: Zwei aus Wolken kommende Arme halten eine gesiegelte Schrift über einer Festung in einer Landschaft. $T$ : Du bist in deinem Übermut zu hoch gestiegen, mußtest vieles aus der Hand geben und wirst nun mit deinen eigenen Waffen geschlagen. Hoffart währt nur eine gewisse Zeit. Dein Herz hat dich verführt; die Schwachen hast du bedrückt und den Nächsten die Freiheit geraubt. Deine Weisheit wurde zur Torheit. K: Selig sind, die armen Geistes sind.

$B X V$ : Eine dreifache Krone, von einem schweren runden Stein (Mühlstein ?) überhöht. Im Hintergrund die strahlende Sonne. T: Selbst das beste muß ohne Haupt verderben. Wer kein Haupt anerkennt, wird hoffärtig. Auch du wirst wieder ein Glied wie andere Glieder werden, um ein gemeinsames Haupt zu tragen. Diese Einigung kommt einmal zustande, vielleicht wenn du schläfst. $K$ : Der Kaiser ist das Haupt; die lutherischen Prädikanten wollen selber Herren sein. Der Schlaf bedeutet Ende und Untergang.

$B X V I$ : Ein Kinderkopf blickt auf zerrissene Bücher und Schriften auf einem Tisch. T: Welcher Erwachsene schämt sich nicht seiner Schulhefte und vernichtet sie nicht? So wird es auch deiner Arbeit ergehen. Man wird erkennen, daß nicht alles Perlen sind, die als Perlen ausgegeben wurden. Eine Hand wird auf dich fallen und dich zerreißen wie einen Fetzen. $K$ : Was in der Schule gelernt wird, sind nur Buchstaben. Es ist nicht alles Gottes Wort, was dafür ausgegeben wird. Im Luthertum wird der Buchstabe für Geist gehalten; an Stelle der Wahrheit werden ihre Zeichen angenommen.

B XVII: Ein Steinmetz behaut eine Platte. T: Wer ein Haus baut, soll es vor dem Feind schützen; wer eines zerstört, dafür sorgen, daß es nicht wiederaufgebaut werden kann. Menschenwerk ist zerbrechlich, auf Sand und nicht auf Felsen gebaut. $K$ : Im Luthertum ist alles auf Buchstaben, Zeremonien und Äußerlichkeiten gegründet, ohne Herz und Geist.

$B X V I I I$ : Der Windhauch aus dem Mund eines aus Wolken kommenden Kopfes wirft drei Säcke um, daß ihr Inhalt herauskollert. T: Ob es dir jetzt schon gut geht und sich alle deine Wünsche erfüllen, so wird doch die Sündflut über dich kommen und ein rauher Mittagwind dich verblasen wie Staub auf der Erde. Nicht Wollust ist unser irdisches Los; die Erde ist ein Jammertal. K: Der Kaiser und Österreich kommen als Mittagswind, die Sündflut als Trübsal.

$B X I X$ : Auf dem Rücken liegt ein zu Fall gekommener Hirsch. T: Statt ruhig zu sein, gefällst du dir in schönen Sprüngen. Bald wirst du zum 
Springen gehetzt werden, bis du zu Fall kommst. Menschenwerk ist eitel, und Übermut führt zu einem schlimmen Ende. Hättest du es bedacht, wärst du nicht zu Fall gekommen. K: Im Luthertum ist keine Weisheit Gottes. Die Lutherischen sind übermütig geworden. Bündnisse sind nur Menschenwerk.

$B X X$ : Ein Mann geht mit umgekehrtem Kreuz über Feld. $T$ : Menschenweisheit, mag sie noch so gefallen, hat keinen Bestand und geht zur Erde, aus der sie gekommen ist. Ein Ding steht nur so lange aufrecht, als man es aufrecht halten kann. Wohl bringt die Zeit Rosen; aber sie verwelken bald. Auch deine Weisheit wird vergehen, weil sie nur aus dir selber stammt. K: Menschenweisheit muß Gottes Weisheit weichen. Du bist aus dir selber und nicht aus Christo. Schriftauslegung nach menschlicher Klugheit hat keinen Bestand.

$B X X I$ : Ein gekrönter Adler stürzt sich auf einen in den Wellen versinkenden Mann. T: Du wirst einem müden Vogel gleich werden. Man wird dich in deinem Nest aufstöbern und du wirst dem weichen müssen, den du vernichten wolltest. Du wirst Nest und Eier verlassen müssen und mit deinen Jungen in Stricke fallen, die du andern gelegt hast. Dein Kleid wird einen andern zieren. $K:$ Du und dein Kurfürst werdet in Wittenberg überfallen werden. In den Stricken, die du Schwärmern und Täufern gelegt hast, wirst du dich verfangen.

$B X X I I$ : Ein Ritter und ein Mönch liegen vor dem Wall einer mauerbewehrten Stadt, gegen die ein Schwert von oben stößt. T: Ritter und Geistliche willst du dir untertan machen. Deine geheimen Pläne sind offenbar geworden. Du denkst: «Wer soll mich hindern?» Es wird aber dein Blut kosten. Bedenke: das Reich ist nicht von dieser Welt. Werde demütig, dann wird dir wohl werden. $K$ : Luthers Hoffart; Kriegsleute und Pfaffen.

$B X X I I I$ : Drei Männer disputieren miteinander auf Kanzeln. Ein vierter schleicht mit einem Sack auf dem Rücken wie ein Dieb weg. T: Die Dreizahl der Gottheit ist in Eins beschlossen, und in dieser Zahl sollen auch die Menschen sein. Jedes in sich gespaltene Reich muß vergehen; kein Reich vergeht ohne innere Spaltung. Der beste Schutz, das sicherste Bündnis eines Reiches ist seine innere Einheit. Wo Spaltung ist, kämpft eins wider das andere, hat nichts Bestand. Könntest du doch ins Eins eingehen! $K$ : Luther hat das Reich gespalten. Gespalten werden kann nur der Buchstabe, nicht der Geist. Eins werden heißt deutsche Einheit in Christo.

$B X X I V$ : Ein sitzender Mann und ein sitzender Bär reden miteinander. Zwischen beiden steht ein Hahn, gegen den ein Schwert von oben gezückt 
ist. $T$ : Ihr verbündet euch umsonst. Nicht mit Menschenverstand sollt ihr regeln wollen, was in Gottes Hand steht. Tut ihr's dennoch, wird doch der über euch herrschen, von dem geschrieben steht: Gebt ihm, was ihm gehört. Euer Rat wird zerstört; Menschenvernunft schafft nichts bleibendes. Nur was aus Gott kommt und geordnet ist, hat Bestand. $K$ : Das Schmalkaldische Bündnis. Menschenvernunft kennt nur den Buchstaben.

$B X X V$ : In den Wolken schwebt ein von Stricken umgarnter Mann: $T:$ Du hast dich in deinen eigenen Zweifeln verfangen. Du hast auf Zweifel und Sand gebaut und wolltest selber Siegel sein, das du nicht bist. Du und die von dir Verführten sind in Trauer gekommen und werden noch mehr weinen. $K$ : Glauben an den Buchstaben statt an den Geist.

$B X X V I$ : Eine Rose auf einer Krone wird von einem F überragt. $T:$ Du bist im Sommer, in der Rosenzeit; aber dein Sommer ist widerwärtig und bringt Spaltung. Was auf Sand gebaut ist, muß vergehen. $K$ : Das $F$ über der Rose bedeutet Friedrich. Der widerwärtige Sommer ist die Zeit des Kriegsbeginns; die Bauten auf dem Sand sind ein Bild für die geschlossenen Bündnisse.

$B X X V I I$ : Fünf aus Wolken kommende, mit Schwertern bewaffnete Arme. T: Wo viele Häupter regieren, geht es übel zu. Daß man das Eins vergessen hat, ist die Ursache aller Spaltungen. Wer, statt zu dienen, seine eigene Gelegenheit sucht, wird nicht ans Ziel kommen. Dem die Wahrung des Eins anbefohlen ist, wird in seinem Bestreben fortfahren, und unter ihm freuen sich alle Gepeinigten und Gedrückten. $K$ : Der Kaiser ist der Wahrer der Einheit; der Unfriede kam aus Geiz und Eigennutz.

$B X X V I I I$ : Männer, um ein Kardinalskreuz sitzend, disputieren. $T$ : Sie werden nicht einig werden und sollten achten auf das kommende 42. Vielleicht etwas vorher, vielleicht etwas nachher wird der kommen, der euch biegen wird, wie es euch nicht gefällt, wie einen Zweig. Im Menschen, der sich absondert, ist keine Weisheit; möchte er doch an seine schwere Rechnung am Tage des Zornes denken. $K$ : Der Mann, der biegt, ist der Kaiser. Luthers Rat ist nicht aus Gott noch aus Christo. Es ist nicht alles Gold, was als solches ausgegeben wird.

$B X X I X$ : Ein mit der Inful geschmücktes Lamm (die Inful als Zeichen der Heiligkeit und Unverletzlichkeit). $T$ : Die Zeit wird kommen, da jedes auf seiner eigenen Weide sein Futter suchen darf, jedes an den Pflug gespannt ist, der ihm anbefohlen ist. In fremder Weide grasen müssen, bringt Jammer, Elend und Zank. Einigkeit wird erst dann sein, wenn jeder in den für ihn bestimmten Stall gehen darf. Nicht weit von 43 entfernt kommt die 
Stunde, da es jedem freisteht, in seiner eigenen Matte zu grasen; wie selig wird diese Stunde sein. K: Glaubensfreiheit; sie werden alle von Gott gelehrt werden und sich ihres Glaubens freuen.

$B X X X$ : Drei disputierende Männer. $T:$ Du konferierst und disputierst, aber du hattest keinen Gegner. Steh doch ab und bedenke, daß alles nur deine eigene Weisheit ist. Du gehörst nicht auf PETrI Stuhl; er wird fallen. Steh ab. Der dein Herr ist, wird dein Fürnehmen vereiteln. K: Die Konferenzen führten zum Schmalkaldischen Bund. Du wärest gern Papst; aber Christus wird dein Herr sein.

$B X X X I$ : Tanzende Kinder, nackt. $T$ : Ums Jahr 60 wird der brüllende Löwe einfallen und viele niederwerfen. Wie wohl wird dann denen sein, die ruhig und fröhlich wie Kinder sein können. Menschenweisheit bringt nur Unruhe und Kümmernis. K: Gottlob, Jahr 60. Der brüllende Löwe ist der Antichrist. Menschenweisheit ist Eigenwissen. An diese Prophezeiung glaube ich standhaft.

$B X X X I I$ : Ein ruhender Mann schläft unter einem Baum. Strahlende Sonne steht am Himmel. Er liegt zu Füßen eines burgbewehrten Hügels. $T$ : Du hast dich hart gemüht, das goldene Zeitalter zu schaffen; drum sollst du von deinem Tagewerk ausruhen. Selig, wer unter dem Schlaf geboren wird; nichts Arges wird er wissen. Du hast mit viel Mühe die Reinigung vollbracht; niemand hat dich überwunden und niemand wird dich wieder aufwecken. $K$ : Christus ist nicht überwunden. Angelorum septimus, die Zeit des siebenten Engels, da das Geheimnis Gottes vollendet wird, wie er es seinen Knechten, den Propheten, verkündigt hat.

In seiner «beschlußred» gibt Paracelsus eine eigene kurze Erklärung seiner Prognostikation. Er wollte in derselben weissagen, in 32 Artikeln, was sich in der Endzeit, in den kommenden 24 Jahren, ereignen und vollenden werde. Der kleinste dieser Artikel bedürfte nach seinem Geständnis eines ganzen Buches. Und wie viel Jammer würde aus jedem zutage treten! Der zweite Artikel zeigt zwar eine Lilie. Sie ist aber nicht das Symbol der Reinheit, sondern nur der Hoffart. Billiger wäre das Bild einer Kröte gewählt, die sich aufbläht vor Gift. Darum wurde auch die Lilie an einem Dornstrauch gezeigt. Die folgenden Bilder sagen viel Not und Zusammenbruch voraus. Verlassen werden von den besten Kampfgenossen, macht schwach und machtlos. Wollte man aber den ins rechte Licht rücken, der «so wol gehalten und kint im Haus ist», wie vieles wäre noch zu berichten, nicht nur Allgemeines, sondern auch Besonderes. Wie viel Not würde sich 
dabei zeigen! Die nächsten Bilder melden den Kampf und Untergang. Sie künden vom Suchen nach fremder Hilfe und Schmieden von Ketten, von Fallen und Brechen, vom Suchen nach Kühlung von der Hitze des Kampfes. Der achte Artikel verkündet den kommenden Sieger; würde der Sieg nicht ihm, Gott, zufallen, könnten auf Erden nie mehr Ruhe und Frieden einkehren. Alle würden sich zersplittern und auseinandergehen, ein jedes nach seinem eigenen Kopf und jedes allein, ohne Wissen darum, warum sie eigentlich auf Erden sind, mit dem dünkelhaften Vorsatz: "Also wil ichs, also thon ichs.» Eine gekrümmte Rute schnellt immer in die alte Richtung zurück; auf die Dauer läßt sich nichts Gekrümmtes bewahren. Wer nicht in seiner eigenen Richtung bleibt, löst sich langsam auf. Das folgende Bild nimmt Bezug auf das Schicksal Ninives, der Stadt, in der alles in Buße und Asche gehen mußte, weil der Herr sich über ihrer Hoffart erzürnt hatte. Er zeigte den Verführten aber doch sein Erbarmen, die nicht zwischen rechts und links, zwischen ihren Rechten und Pflichten hatten unterscheiden können. Das elfte Bild zeigt einen Büßenden, der an seiner eigenen Pfote saugt, ein trauriges Los; was sich einer aber eingebrockt hat, muß er eben auch ausessen. Gott selber hat Macht und Herrschaft auf Erden bestimmt und der Mensch soll sich darunter beugen, den Machtstuhl anerkennen. Die 13. Figur ermahnt den Übermütigen zur Demut. Selig sind die Armen; sie stehen in Gottes Hand und werden nicht verlassen werden. Buchstaben sind kein genugsamer Schutz; welcher Buchstabe könnte nicht durchlöchert werden? Menschen können noch so viel schreiben, noch so viel bauen an ihrem Turm; das Dach muß doch von höherer Hand gesetzt werden, über welches niemand hinausbauen darf. Der Stein muß wieder dorthin gelegt werden, woher er genommen wurde, da sonst höchstens ein babylonischer Turm entsteht. Nur der wahrhaft weise Mann kennt das ihm gesteckte Ziel. Vor Gott ist alle menschliche Stärke einem Rohr gleich. Wer sein eigen Haus nicht sauberhalten kann, wie sollte er berufen sein, anderer Häuser zu reinigen? Die Unzufriedenen werden abspringen und die unruhebringenden Bündnisse zerbrechen. Die folgenden Artikel zeigen, wie sich alles wieder nach Ruhe und Friede zurücksehnt, die weitern Bilder, wie an menschlicher Weisheit nichts gelegen ist und der Mensch nichts erreden kann. Der Zweifel ist des Glaubens Feind; dieser muß auf Felsen gegründet sein. Der Mensch kann nichts erdenken; nur eine höhere Erleuchtung kann ihm Gewißheit geben. Weder Bündnisse noch Ketten können so stark sein, um zu verhindern, daß am Ende jeder dort grase, wo seine Weide ist, an seinem eigenen Pflug gespannt werde. Hilfe und Errettung wird nur denen 
zuteil, die wie Kinder ohne List und Tücke sind. Gott hat schon oft wieder Ruhe gemacht und ist selber wieder Ruhe geworden. Wenn er aber erweckt wird und sich aufrichtet, dann erzittern alle Kreaturen. Er kehrt um und richtet, wie es ihm gefällt, und zu diesem Werk hat er sich noch 24 Jahre vorgenommen, Jahre, die vor ihm sind wie ein Tag. Er wird nicht Artikel um Artikel wegschaffen, sondern seine Taten bis aufs Ende aufsparen. Wer mag wissen, wer gemeint ist? «Darumb so mag niemants genent noch in argwon genomen werden bis alles für ist, dan also sol es ein.»

Die Randnotizen besagen, daß da einer Lehrer sein wollte ohne Auftrag. Auf seiner eigenen Matte kann nur der Wiedergeborene weiden. Christus ist es, der alles umkehrt nach seinem Wohlgefallen.

Paracelsus lehrt in dieser Schrift, daß nur Menschen, die wie Kinder fromm und fröhlich sind, ins einige Reich, ins Reich des Heiligen Geistes eingehen können, das Gottes ist, und daß nur einem die Einheit des Reichs auf Erden anvertraut ist, dem Kaiser. Mit Menschenwitz kann nichts Dauerhaftes geschaffen werden; er bringt nur Spaltung zustand und Unordnung. Den Menschen ist von höherer Hand eine Ordnung gesetzt, ein Haupt, das sie alle als Glieder tragen sollen. Ein Mann wollte mit löblicher Absicht Ordnung herstellen. Da kam der Übermut über ihn, die Hoffart, da ihm zuerst vieles glückte. Er anerkannte kein Haupt mehr über sich, wollte nicht mehr Glied, sondern selber Haupt sein und fand Kampfgenossen, die ihm zujubelten. Bald wollten aber auch sie sein Haupt nicht mehr anerkennen, sondern selber ein solches sein, so daß alle auseinanderliefen, ein jeder seinen eigenen Weg. Um die Macht zu erhalten und zurückzugewinnen, schloß er Bündnisse, legte er Ketten und Stricke, um die Herde beieinanderzuhalten. Als er den obersten Stuhl besteigen wollte, fiel dieser um, weil er ihn nicht tragen wollte. Er verfing sich in den Stricken, die er andern gelegt hatte. Alle seine menschliche Macht zerbrach wie auch die derer, die ihn hätten schützen sollen. Und schließlich erreichte ihn sein Gericht. Denn Gott erzürnte sich über seinen Übermut, wie er sich über den frevelhaften Übermut Ninives erzürnt hatte. Das Gericht kam über ihn und seine Verführten, von dem nur die verschont blieben, die wie Kinder ohne List und Tücke waren. In 24 Jahren hat der Herr der Herren Ruhe und Ordnung wiederhergestellt. So sah für Paracelsus diese Endzeit aus, die er weissagte. Wen anders als Luther könnte er wohl gemeint haben mit den von ihm Verführten ? Er selber sagt freilich, niemand könne wissen, wen diese Weissagung meine, und keiner solle in Argwohn genommen werden; jeder sehe nur die Fehler des andern und versuche, sich selber zu 
beschirmen. Sollte nicht am Ende auch Paracelsus versucht haben, sich «selbs zu beschirmen»? Behaften konnte man ihn bei dieser Prognostikation nicht.

Wenn Luther mit dieser Weissagung wirklich gemeint war, hat sich Theophrast mit derselben geirrt? Klingt es nicht wie eine Bestätigung, was des Wittenbergers Biograph, Adolf Hausrath, am Schluß seines zweiten Bandes aussagt? «Luthern selbst aber war über den traurigen Zänkereien der letzten Jahre die Erkenntnis gekommen, daß ein Geschlecht von dogmatischen Streithähnen, Klopffechtern und unnützen Spektakelmachern erwachsen sei, das die schwersten Kämpfe für die Zukunft erwarten lasse. Dieser Nation, in der jeder eigenwillig seinen besondern Weg einschlug, war nicht zu helfen ... Eine Zeit der Not und des Verderbens schien sich ihm für sein Deutschland vorzubereiten, in dem eine Hand wider die andere war. Er selbst durfte noch in Frieden die müden Augen schließen, dann aber krachten die Geschütze des Spaniers, und der Herr rief vom Himmel: Für ein ungehorsam Volk habe ich meine Wunder nicht getan. Da ihr nicht hören wollet, so sollt ihr fühlen.» Das war die Situation des Jahres 46; hat Paracelsus nicht auffallend klar den Gang der Ereignisse vorausgesehen? Luther hätte er freilich die Frage gestellt, ob er wirklich nicht wisse, warum denn alles so gekommen sei; er konnte diese Frage damals nicht mehr stellen, weil seine irdische Wanderschaft 1541 zu Ende gegangen war.

Wie man sich auch zu Theophrasts Prognostikation und diesem Deutungsversuch stellen mag, wird man auf keinen Fall verkennen können, daß es sich um eine ausschließlich religiöse Weissagung handelt, aus eschatologischer Sicht heraus. SUDHOFF hat sie zu Unrecht in die naturwissenschaftlich-philosophische Reihe seiner Gesamtausgabe der Werke des Hohenheimers aufgenommen; hier ist nicht ihr richtiger Platz. Die religiösen und religionsphilosophischen Voraussetzungen seiner Arbeit sind in der Vorrede klar umschrieben. Paracelsus ist Spiritualist. Sehnsüchtig harrt er wie einst die Joachimiten auf den Anbruch des dritten Reiches, des Reichs des Heiligen Geistes, die Zeit, da es nur noch eine Herde und einen Hirten geben wird, nur noch ein Reich und ein Volk, alle geeint im großen Eins, im Heiligen Geist, der das Haupt aller Glieder, aller Nationen und Menschen sein wird. Buchstaben und Gesetz werden dann überwunden sein; die Zeiten der Spaltung, tempora nationum, gehen ihrem Ende entgegen, aber nicht ohne Kampf, nicht ohne gerechtes Gericht über die hoffärtigen Spalter und Verführer. 
Wieder ist Theophrast, der sich ewig nach Heimat sehnende heimatlose einsame Wanderer, der außer alle nationale, soziale, berufliche, philosophische, medizinische und religiöse Bindung und Ordnung gestellte Seher der Verkünder einer ewigen, unwandelbaren Ordnung, wie im Volumen Paramirum, wie im Opus Paragranum. In der von Gott gesetzten Ordnung für Mensch und Natur, für alles Kreatürliche, ist Gesundheit, Ruhe und Frieden begründet; in der Störung dieser Ordnung liegt der Grund für Krankheit, Zank, Streit und Untergang. Seine religiösen und seine ärztlichen Einsichten stammen aus derselben Quelle und können nicht voneinander getrennt werden. Alle Einsichten werden geoffenbart; das Licht der Natur erleuchtet aber nur den, der im Licht des Herrn wandelt. So steht auch die Prognostikation durchaus in einer Reihe mit den Werken der Para-Zeit. Sie ist die direkte Fortsetzung seiner Weissagung im Kometenbüchlein vom Jahr 1531. Damals zeigte ihm der Komet an, daß der Herr nun selber das Schicksal von Kaiser und Reich in die Hand nehme. Es habe sich ein Teil wider den andern erhoben; Gott werde aber die Einheit seines Reiches wieder herstellen. "Alsdan gon die zeichen an und got bricht das, das im nit gfellig ist und das der mensch an im selbs nit hat mögen wenden, so wendet got es, diewil er die sinen nit verlaßt.» Es waren aber nur Zeichen, die den Anfang der «operation» verkündeten, die jetzt, 1536, in 24 Jahren zu Ende gehen sollte.

Theophrasts Prognostikation steht in ihrer Zeit keineswegs vereinzelt. Noch war die Weissagung der Joachimiten lebendig, die den Beginn des dritten Reiches aufs Jahr 1260 erwartet hatten. Unser Exemplar mit den Randnotizen ist zusammengebunden mit einem Büchlein aus dem Jahr 1608 mit dem Titel Der von Gott bestimpten Zahlen des Antichrists/1260 endlicher Aussgang und Ende, ohne Angabe von Verfasser, Verleger und Druckort. Joachimitischer Geist tritt uns auch aus Theophrasts Weissagungen entgegen. Zur Zeit, als er seine Schrift niederschrieb, erwartete noch immer Melchior Hofmann hinter vergitterten Gefängnisfenstern in Straßburg den Anbruch der von ihm fürs Jahr 1533 vorausgesagten Weltenwende. In Luther sah er den Antichrist der Endzeit. Wie eine Antwort an HoFMANN lesen sich die Worte Theophrasts: «In der operation ist noch kein end, waren nur anfeng. Es ist aber jetz die zeit das si zum end gehen werden.» 1536 hielt sich gleichzeitig mit Paracelsus auch Sebastian Franck in Ulm auf, der zwei Jahre später beim selben Verleger Heinrich Steiner in Augsburg seine Guldin Arch veröffentlichte. Ihn bedrängten damals ähnliche Gedanken und Probleme wie den Hohenheimer, der schon mehr- 
mals seinen Weg gekreuzt hatte; auch er sehnte sich nach der Überwindung von Gesetz und Buchstaben durch den Heiligen Geist. Und solcher gab es noch viele. Manchem graute es, daß man versuchte, mit Menschensatzungen und menschlicher Macht etwas zu erzwingen, das nur aus dem Geist geboren werden konnte. Schrieb nicht auch GRYNäUs von Basel aus an LEo Jud nach Zürich: «Wir haben die Zeremonien abgeschafft, und das Evangelium behauptet nicht durch Geduld und Langmut, sondern durch die Stimmen der Mehrheit, so daß es auf Menschenhoffnung und Menschenbeifall beruhte, nicht auf den rechten Wurzeln ... Mit ungeheurem Selbstvertrauen haben wir uns zur Entscheidung durch die Waffen entschlossen. Nicht durch Gewalt und Schrecken, durch Demut und Liebe müssen wir die Wahrheit aufrechthalten.» Man sieht, Paracelsus stand mit seinen Bedenken nicht allein. Manch anderer wäre noch zu erwähnen, und wie viele werden gleiches bedacht haben, sicher nicht nur Täufer und Schwärmer, nicht nur Ketzer. Noch waren die Fronten nicht erstarrt.

So sehr aber die Prognostikation von 1536 eine Weiterführung der Weissagung des Kometenbüchleins von 1531 darstellt, so wenig kann man einen tiefen Unterschied übersehen, der in der Dedikation der beiden Schriften liegt. Die Kometenschrift war Leo Jud und UlRich Zwingli gewidmet, die Prognostikation dem römischen König und spätern Kaiser FerdiNAND II., dem Fürsten, der mit eiserner Hand den neuen Glauben in seinen österreichischen Erblanden ausgerottet hatte, einem energischen Bekämpfer des Schmalkaldischen Bundes, der andererseits vor allem als Kaiser den Religionsfrieden zu wahren und die Reichseinheit zu erhalten wußte, die Einheit des heiligen römischen Reiches deutscher Nation, die wie die Glaubenseinheit auch Paracelsus' Herzensangelegenheit war. Seine Einstellung zu den Neugläubigen hatte sich von Grund auf geändert. 1531 noch empfahl er seine Kometenschrift den beiden Zürchern zum eingehenden Studium, gleich wie er auch ihre Werke mit aller Aufmerksamkeit lese. Die Zwischenzeit hatte den Kappelerkrieg und das Schmalkaldische Bündnis gebracht, und was reformatorische Bewegung gewesen war, erstarrte immer mehr zu neuen Maurerkirchen mit obrigkeitlicher Gesetzlichkeit. Jetzt sah Paracelsus in Ferdinand den Mann, dem die Reichseinheit anbefohlen war, als ihm gestellte Aufgabe. Noch war ja KARL V. Kaiser. Auch hier ist es erstaunlich, wie richtig ThEOPHRAST die realen Gegebenheiten einschätzte, mußte doch Ferdinand diese Funktionen später teilweise gegen seinen kaiserlichen Bruder erfüllen. Es geht Theophrast keineswegs um einzelne Glaubenssatzungen, allein um das große Eins. Dieses erwartete er nicht von 
der reformatorischen Bewegung, sondern vom Kaiser, dem römischen König in des Kaisers Namen.

Und nun nochmals der Kommentator. Wir wissen nicht, wer er war, nicht genau, wann er gelebt hat; seine Schrift gehört dem 16. Jahrhundert an. Er war ein studierter Mann; denn er verstand das Latein. Er kannte auch die Bibel und gehörte sicher zu den Spiritualisten, hoffte er doch «standhaft» auf die Erfüllung der Prophezeiung, die er nicht als Kritiker, sondern als andächtig Hörender vernahm. Und doch wären kritische Bemerkungen leicht möglich. Fast unmerklich tritt im Lauf von TheopHrasts Weissagung an die Stelle von Babel Ninive, an Stelle einer Weltenwende eine Episode, an Stelle eines alle Völker der Erde umspannenden Geschicks deutsches Schicksal. Ein Mann hat in Deutschland durch seinen Hochmut und seine Vermessenheit Spaltung, Unruhe und Streit herbeigeführt, bis Gott aus seiner Ruhe sich erhob, um die Ordnung wiederherzustellen, unter Bestrafung der Schuldigen; nachher geht er wieder in die Ruhe ein. Der Kommentator hat davon offenbar nichts bemerkt. Er kritisiert nirgends; er will nur hören und verstehen. Manches hat er wohl falsch verstanden. Das F, das im 26. Bild die Rose auf der Krone überhöht, ist kaum FriedRIGH. Was liegt daran. Daß er das Ganze wahrscheinlich recht verstanden hat, wer kann sich dieser Einsicht entziehen ? Gerade als Spiritualist, der den Gedankengängen von Theophrast nahe stand, war er einem Verständnis von dessen Weissagung besonders geöffnet. Ist seine Auslegung die einzig mögliche? Auf diese Frage kann der Schreiber dieser Zeilen nur mit Theophrasts eigenen Worten antworten:

«Andere müssens baß erkennen denn ich, auch verstehen, wen es berühre.» 